PROCEEDINGS OF THE

AMERICAN MATHEMATICAL SOCIETY

Volume 137, Number 7, July 2009, Pages 2403-2406

S 0002-9939(09)09847-5

Article electronically published on February 24, 2009

\title{
CONFORMALLY STATIONARY LORENTZIAN TORI WITH NO CONJUGATE POINTS ARE FLAT
}

\author{
FRANCISCO J. PALOMO AND ALFONSO ROMERO \\ (Communicated by Jon G. Wolfson) \\ Dedicated to Professor A. M. Naveira on his 68th birthday
}

\begin{abstract}
A Lorentzian torus which admits a timelike conformal vector field and with no conjugate points on its timelike and spacelike geodesics is proved to be flat. If only the absence of conjugate points on timelike geodesics is assumed, a counterexample is shown.
\end{abstract}

\section{INTRODUCTION}

A Lorentzian manifold which admits a timelike conformal (resp. Killing) vector field is called conformally stationary (CS) (resp. stationary). In 9 it is shown that a compact CS Lorentzian manifold is geodesically complete (a compact Lorentzian manifold may be geodesically incomplete; see for instance [7, 8] to get many examples of incomplete Lorentzian tori). Any CS Lorentzian torus $\left(\mathbb{T}^{2}, g\right)$ is (globally) conformally flat; in fact, the metric $(1 /(-g(K, K))) g$, where $K$ is a timelike conformal vector field on $\left(\mathbb{T}^{2}, g\right)$, is flat. Conversely, a conformally flat Lorentzian torus admits a timelike conformal vector field [8]. Thus, to the contrary of the Riemannian case, a Lorentzian torus may be non-conformally flat.

A remarkable theorem by E. Hopf in 1948 showed that a Riemannian 2-torus with no conjugate points is flat [6] (the result remains true for higher dimensions [2]). The technique used by Hopf cannot be adapted to the Lorentzian case. However, several integral inequalities, involving the Ricci tensor, for a compact CS Lorentzian manifold have been proved and shown to be useful for studying conjugate points on null geodesics [4, 5]. These inequalities have been obtained by making use of a new technique based on the geometry of the Lorentzian manifold called the null congruence manifold 4. Starting from an arbitrary time-orientable Lorentzian manifold and one of its timelike vector fields, the null congruence is constructed restricting the Sasaki metric on certain codimension-two submanifolds of the tangent bundle. When the timelike vector field is conformal, the null congruence manifold possesses several crucial properties, which makes it useful to analyze the behavior of conjugate points on null geodesics in the subfamily of compact CS Lorentzian

Received by the editors June 13, 2008.

2000 Mathematics Subject Classification. Primary 53C50, 53C22, 53C25.

Key words and phrases. Conformally stationary Lorentzian torus, conjugate points, flat Lorentzian torus.

Both authors were partially supported by the Spanish MEC Grant MTM2007-60731 with FEDER funds and the Junta de Andalucía Regional Grant P06-FQM-01951.

(C)2009 American Mathematical Society 2403

Reverts to public domain 28 years from publication 
manifolds 4, [5]; in particular, even another proof of the classical Hopf theorem was given [5].

In the 2-dimensional case, there exists no conjugate point on a null geodesic 1, Lemma 10.45], and the null congruence technique does not work. However, in [5] we introduced a procedure to deal with conjugate points on non-null geodesics of Lorentzian surfaces that we will use also in this paper (see proof of Theorem 3.1). Both the previously explained nice properties of CS Lorentzian tori and clear technical reasons lead one to conjecture a natural extension of the Hopf theorem to the family of CS Lorentzian tori. Thus, the main aim of this paper is to prove the following result (Theorem 3.5):

\section{If a CS Lorentzian torus has no conjugate points along its geodesics, then it is flat.}

Moreover, in Counterexample 3.6, a CS Lorentzian torus with no conjugate points on its timelike (or spacelike) geodesics which is non-flat is constructed. Therefore, Theorem 3.5 cannot be generalized under a weaker assumption.

\section{Preliminaries}

Let $(M, g)$ be an $n(\geq 2)$-dimensional Lorentzian manifold, that is, a (connected) smooth manifold $M$ endowed with a non-degenerate metric tensor $g$ with signature $(-,+, \ldots,+)$. We shall write $\nabla$ for its Levi-Civita connection, Ric for its Ricci tensor, $S$ for its scalar curvature and $d V$ for the canonical measure defined by $g$. Recall that the vector field $K$ is said to be conformal when $\mathfrak{L}_{K} g=2 \rho g$ with $\rho \in C^{\infty}(M)$. If $\rho=0, K$ is said to be Killing. If $(M, g)$ is a CS Lorentzian manifold and $K$ is a timelike conformal vector field $K$, we put $h=1 / \sqrt{-g(K, K)}$ and write $U=h K$ for the normalized vector field obtained from $K$.

Conjugate points on null geodesics of a compact CS Lorentzian manifold have been studied in [4, [5]. In particular, the absence of such conjugate points provides the following integral inequality [5, Corollary 4.3].

If an $n(\geq 3)$-dimensional compact CS Lorentzian manifold $(M, g)$

has no conjugate points on its null geodesics, then

$$
\int_{M}[n \operatorname{Ric}(U, U)+S] h^{n} d V \leq 0
$$

where $K$ is the timelike conformal vector field and $U=h K$. Moreover, equality holds if and only if $(M, g)$ has constant sectional curvature $c \leq 0$.

\section{MAIN RESUlts}

Theorem 3.1. If a CS Lorentzian torus $\left(\mathbb{T}^{2}, g\right)$ possesses no conjugate points along its timelike geodesics, then for any integer $m \geq 3$ we have

$$
\int_{\mathbb{T}^{2}} G h^{m} d A \geq 0
$$

where $G$ denotes the Gauss curvature and $d A$ the area element of $\left(\mathbb{T}^{2}, g\right)$. Moreover, if the equality holds for some $m$, then $\left(\mathbb{T}^{2}, g\right)$ must be flat.

Proof. Let $N^{k}$ be a $k(\geq 1)$-dimensional compact flat Riemannian manifold. Using the form of the geodesics in a semi-Riemannian product we conclude that the Lorentzian manifold $\mathbb{T}^{2} \times N^{k}$ has no conjugate points along its null geodesics. On 
the other hand, $K$ induces a timelike conformal vector field on this semi-Riemannian product. Taking into account the expressions for the Ricci tensor and the scalar curvature of $\mathbb{T}^{2} \times N^{k}$, the integral inequality (11) gives $\int_{\mathbb{T}^{2}} G h^{2+k} d A \geq 0$, for every $k \geq 1$. The equality holds if and only if $\mathbb{T}^{2} \times N^{k}$ has constant sectional curvature, that is, if and only if $\left(\mathbb{T}^{2}, g\right)$ is flat.

An alternative expression for (2) can be obtained from the following result.

Proposition 3.2. On any CS Lorentzian torus $\left(T^{2}, g\right)$ we have

$$
\int_{\mathbb{T}^{2}} G h^{m} d A=-m \int_{\mathbb{T}^{2}} h^{m+4} g\left(\nabla_{K} K, \nabla_{K} K\right) d A,
$$

where $m$ is any non-zero integer.

Proof. Since the metric $h^{2} g$ is flat, we know $G=\triangle \log h$, where $\triangle$ denotes the D'Alembertian operator of $g$. Therefore the divergence theorem shows that

$$
\int_{\mathbb{T}^{2}} G h^{m} d A=-\int_{\mathbb{T}^{2}} g\left(\nabla h^{m}, \nabla \log h\right) d A .
$$

The gradients in the right-hand member can be expressed as

$$
\nabla h^{m}=m h^{m+2}\left(2 \rho K-\nabla_{K} K\right) \text { and } \nabla \log h=h^{2}\left(2 \rho K-\nabla_{K} K\right),
$$

and so the result easily follows.

Let $(\Sigma, g)$ be an oriented and time-oriented Lorentzian surface and $K$ be an arbitrary timelike vector field. There is a unique spacelike vector field $E$ such that $g(E, K)=0, g(E, E)=-g(K, K)$ and $\left\{K_{p}, E_{p}\right\}$ is an oriented basis at every $p \in \Sigma$.

Lemma 3.3. Let $(\Sigma, g)$ be an oriented Lorentzian surface. Assume $K$ and $E$ are vector fields on $\Sigma$ as above. Then $K$ is conformal if and only if $E$ is conformal.

Proof. Consider the function $\phi=g\left(\nabla_{K} E, K\right) / g(K, K)$. It is not difficult to compute that $\phi=g\left(\nabla_{E} E, E\right) / g(E, E)$ and then $\left(\mathfrak{L}_{E} g\right)(X, Y)=2 \phi g(X, Y)$ for every $X, Y \in \mathfrak{X}(\Sigma)$. Replacing $g$ by $-g$ the converse follows.

Remark 3.4. It should be noted that the correspondence stated in Lemma 3.3 cannot be restricted to Killing vector fields. In fact, for a warped product metric $-d t^{2}+f(t)^{2} d x^{2}$ on $I \times \mathbb{R}$, with $f$ non-constant, the proper conformal vector field $K=f(t) \partial_{t}$ gives $E=\partial_{x}$, which is Killing.

Now, we are ready to prove the main result of this paper.

Theorem 3.5. If a CS Lorentzian torus $\left(\mathbb{T}^{2}, g\right)$ has no conjugate points along its geodesics, then $\left(\mathbb{T}^{2}, g\right)$ is flat.

Proof. Both Lorentzian metrics $g$ and $-g$ have no conjugate points along their timelike geodesics. Making use of Lemma 3.3 we arrive then at the equality in (2). Therefore, the result follows from Theorem 3.1 .

Counterexample 3.6. A CS Lorentzian torus with no conjugate points along its timelike geodesics is not necessarily flat. In fact, choose a (non-constant) positive periodic function $f: \mathbb{R} \rightarrow \mathbb{R}$, and construct the corresponding CS Lorentzian torus from the warped metric $g=-d t^{2}+f(t)^{2} d x^{2}$ on $\mathbb{R}^{2}$. As shown in [3], this torus has no conjugate points along its timelike geodesics. The Gauss curvature of this torus at the point defined by $(t, x)$ is $f^{\prime \prime}(t) / f(t)$; therefore this torus is not flat. On the 
other hand, if $f^{\prime}\left(t_{0}\right)=0$ and $f^{\prime \prime}\left(t_{0}\right)>0$, then the spacelike geodesic on the torus obtained by projection of $\gamma(s)=\left(t_{0}, s\right)$ has conjugate points. Finally, replacing $g$ by its opposite, we get a non-flat stationary torus with no conjugate point on its spacelike geodesics and with conjugate points on some of its timelike geodesics.

Under the stronger assumption that $K$ is Killing we get

Theorem 3.7. If a stationary Lorentzian torus $\left(\mathbb{T}^{2}, g\right)$ has no conjugate points along its timelike geodesics, then it must be flat.

Proof. Since $K$ is Killing, $g\left(\nabla_{K} K, K\right)=0$ and therefore $\nabla_{K} K$ is spacelike. The equality in (2) is now achieved using Proposition 3.2, and this ends the proof.

Remark 3.8. (a) In [10] all Lorentzian tori with a non-trivial Killing vector field are characterized and explicitly obtained. (b) Theorem 3.7 was already obtained in [5. Now, we give here a different proof.

\section{ACKNOWLEDGMENT}

The authors are thankful to the referee for his/her deep reading and for making suggestions towards the improvement of this paper.

\section{REFERENCES}

[1] J.K. Beem, P.E. Ehrlich and K.L. Easley, Global Lorentzian Geometry, Second edition, Pure and Applied Math., 202, Marcel Dekker, 1996. MR1384756 (97f:53100)

[2] D. Burago and S. Ivanov, Riemannian tori without conjugate points are flat, Geom. Funct. Anal., 4 (1994), 259-269. MR1274115 (95h:53049)

[3] J.L. Flores and M. Sánchez, Geodesic connectedness and conjugate points in GRW spacetimes, J. Geom. Phys., 36 (2000), 285-314. MR.1793013 (2001g:58024)

[4] M. Gutiérrez, F.J. Palomo and A. Romero, A Berger-Green type inequality for compact Lorentzian manifolds, Trans. Amer. Math. Soc., 354 (2002), 4505-4523. (Erratum Trans. Amer. Math. Soc., 355 (2003), 5119-5120). MR1926886 (2003h:53098). MR1997597 (2004g:53076)

[5] M. Gutiérrez, F.J. Palomo and A. Romero, Lorentzian manifolds with no null conjugate points, Math. Proc. Camb. Phil. Soc., 137 (2004), 363-375. MR2092065 (2005g:53124)

[6] E. Hopf, Closed surfaces without conjugate points, Proc. Nat. Acad. Sci. U.S.A., 34 (1948), 47-51. MR.0023591 (9:378d)

[7] A. Romero and M. Sánchez, On completeness of compact Lorentzian manifolds, Geometry and Topology of Submanifolds VI, World Scientific, 1994, 171-182. MR1315099 (96c:53106)

[8] A. Romero and M. Sánchez, New properties and examples of incomplete Lorentzian tori, J. Math. Phys., 35 (1994), 1992-1997. MR.1267937 (95h:53093)

[9] A. Romero and M. Sánchez, Completeness of compact Lorentz manifolds admitting a timelike conformal Killing vector field, Proc. Amer. Math. Soc., 123 (1995), 2831-2833. MR 1257122 (95k:53075)

[10] M. Sánchez, Structure of Lorentzian tori with a Killing vector field, Trans. Amer. Math. Soc., 349 (1997), 1063-1080. MR1376554 (97f:53108)

Departamento de Matemática Aplicada, Complejo Tecnológico, Universidad de MÁlaga, 29071-Málaga, Spain

E-mail address: fjpalomo@ctima.uma.es

Departamento de Geometría y Topología, Facultad de Ciencias, Universidad de Granada, 18071-Granada, Spain

E-mail address: aromero@ugr.es 